\title{
BMJ Open Tobacco-stained fingers: a clue for smoking-related disease or harmful alcohol use? A case-control study
}

\author{
Gregor John, ${ }^{1}$ Sephora Pasche,${ }^{1}$ Nicole Rothen, ${ }^{1}$ Alexia Charmoy, ${ }^{1}$ \\ Cécile Delhumeau-Cartier, ${ }^{2}$ Daniel Genné ${ }^{1}$
}

To cite: John G, Pasche S, Rothen N, et al. Tobaccostained fingers: a clue for smoking-related disease or harmful alcohol use? A case-control study. BMJ Open 2013;3:e003304. doi:10.1136/bmjopen-2013003304

- Prepublication history for this paper is available online. To view these files please visit the journal online (http://dx.doi.org/10.1136/ bmjopen-2013-003304).

During the study, except Cécile Delhumeau-Cartier, all authors were working in the Department of Internal Medicine, Hôpitaux Neuchâtelois, La Chaux-deFonds, Switzerland.

Received 27 May 2013 Revised 28 July 2013 Accepted 9 October 2013

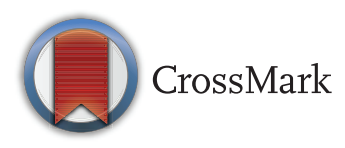

${ }^{1}$ Department of Internal Medicine, Hôpitaux Neuchâtelois, La Chaux-deFonds, Switzerland

${ }^{2}$ Department of Internal Medicine, Geneva University Hospitals (HUG), Geneva, Switzerland

Correspondence to Dr Gregor John; Gregor.John@hcuge.ch

\section{ABSTRACT}

Objective: Tobacco stain on fingers is frequent. However, there is scarce description of this clinical sign. We aimed to explore tobacco stain on fingers as a marker of tobacco-related disease independent of cumulative tobacco exposure, and to find behavioural and environmental characteristics associated with those stains.

Design: Case-control study.

Setting: A Swiss community hospital of 180 beds. Participants: 49 adults presenting tobacco-tars staining on fingers were matched to 49 control smokers by age, gender, height and pack-year (PY).

Outcome measures: Documented smoking-related carcinoma, ischaemic heart disease, peripheral arterial disease, stroke and chronic obstructive pulmonary disease (COPD), also determined by lung function, were compared between groups. Association between harmful alcohol use, mental disorders or unemployment and tarstaining was adjusted for smoking behaviour through conditional logistic regression.

Results: Overall cigarette-related disease was high in the case group (84\%), and symptomatic peripheral arterial disease was more frequent compared to controls ( $O R$ 3.5, CI 95\% 1.1 to 14.6). Smoking-related carcinoma, ischaemic heart disease, stroke and COPD were not statistically different for control smokers. Harmful alcohol use was strongly associated with stains and this association persists after adjustment for smoking unfiltered cigarettes, smoking more than one pack of cigarettes in a day and age at smoking onset (adjusted OR 4.6, CI 95\% 1.2 to 17.2). Mental disorders and unemployment were not statistically significant.

Conclusions: Patients with tobacco-tar-stained fingers frequently have cigarette-related disease, however statistically not more than control smokers matched for PY, except for symptomatic peripheral arterial disease. This study suggests a link between stained fingers and addictive behaviour or concomitant high alcohol consumption.

\section{INTRODUCTION}

The yellow finger stain is an emblem of deeper degradation and enslavement than the ball and chain.

\section{ARTICLE SUMMARY}

Strengths and limitations of this study

- This case-control study explores an easily identifiable and frequent clinical sign among smokers and appreciates the additional risk for health due to tar deposition (influenced by puffing or cigarette characteristics) not included in the usual measure of tobacco exposure (pack-year).

- Diagnoses were obtained from medical records or from patients self-reporting, subject to recall bias.

- Problematic alcohol consumption was not measured with established instruments (eg, Alcohol Use Disorders Identification Test (AUDIT)).

- The sample size was small.

- This study included only the hospital population.

Henry Ford, The Case Against the Little White Slaver (Detroit: 1914).

Tobacco use is the leading cause of preventable death around the world. The 2011 WHO report on the global tobacco epidemic predicted that if current trends persist, more than eight million people worldwide will die from tobacco-related disease each year by 2030. ${ }^{1}$ Although cigarette smoking is by far the most common risk factor for chronic obstructive pulmonary disease (COPD), it is strongly associated with cardiovascular disease, and is responsible for $30 \%$ of all cancer deaths; additional factors are involved in determining each individual's susceptibility to tobacco-related disease. ${ }^{2}{ }^{3}$ Identifying smokers at a higher risk of developing disease could improve their management and screening programmes. ${ }^{4}$

Smoking has been linked to many skin conditions for more than 150 years and evidence exists that skin involvement might be a conspicuous marker of other tobacco-related disease. ${ }^{5}$ For example, smoking-related wrinkles have been associated with a decrease in lung function independent of tobacco 
exposure. $^{6}$ The 'yellow rounded digit' and 'Harlequin nail' signs are two other simple clinical signs which aid the diagnosis of health conditions. ${ }^{78}$ The former, characterised by fingers that are tobacco-stained as well as clubbed, raises the suspicion of lung carcinoma. The latter is seen after an acute illness that causes cessation of cigarette consumption resulting in a bicolour nail (the distal end is cigarette stained, contrasting the newly growing proximal part). We hypothesised that tar deposition itself (figure 1) could be a marker of tobacco-related disease independent of cumulative tobacco exposure. A deeper puff could result in higher tar deposition on the skin and greater inhalation of tobacco, putting the lung and distant organs at increased health hazard.

Tobacco stains on fingers are commonly seen among smokers, but there is scarce description of this phenomenon. Apart from cigarette characteristics, behavioural and environmental factors may be important in stain development. Epidemiological studies worldwide show a higher rate of smoking among people suffering from mental or substance use disorders. ${ }^{9} 10$ Yellow-stained fingers, often observed in psychiatric units and among young drug users (autopsy series), support a possible association between those conditions and tar deposition. ${ }^{11} 12$

We conducted the present study to determine whether tar deposit is an independent marker of tobacco-related disease and to identify factors associated with stain development, such as alcohol consumption, psychiatric conditions, unemployment and smoking habits.

\section{METHODS}

\section{Study design and population}

We performed a case-control study between March 2006 and January 2010 in a 180-bed community hospital in La Chaux-de-Fonds, Switzerland. Eligible patients were adult current smokers admitted to the departments of internal medicine or surgery, or the intensive care unit,

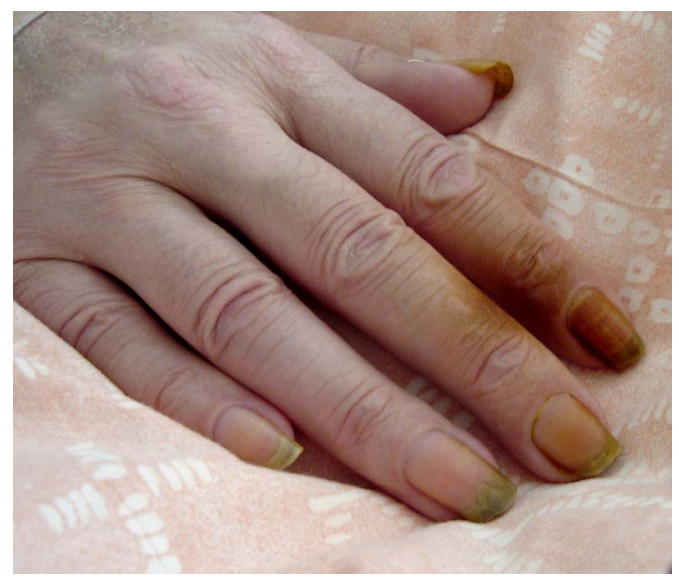

Figure 1 Tar staining seen in a 70-year-old male smoker (80 pack-years). irrespective of the diagnosis or cause of admission. We excluded patients unable to provide informed consent, those post-thoracic surgery or suffering from an earnose-throat (ENT) condition which could prevent lung function testing, patients receiving palliative care and pregnant women. Cases were defined by the presence of tobacco stains on their fingers and were reported to the study group by the medical team or identified by the group through frequent screening. They were included consecutively to avoid selection bias. Controls were selected randomly from the same population to fulfil the matching criteria. All participants provided written informed consent.

\section{Measures and outcomes of interest}

Four physicians dedicated to the study collected all information. The main investigator checked data accuracy using the study information and medical charts. A single 30 min standard interview was performed for each participant to obtain a medical history, a physical examination and to perform pulmonary function tests. Investigations were performed at least 1 month after an exacerbation of a chronic pulmonary condition, pneumonia or acute heart failure. Forced expiratory volume in $1 \mathrm{~s}\left(\mathrm{FEV}_{1}\right)$ and the forced vital capacity $(\mathrm{FVC})$ were assessed using a Microloop spirometer (Micro Medical Limited, England).

\section{Tobacco-related disease}

A history of a tobacco-related disease-ischaemic stroke (haemorrhagic or cardioembolic events excluded), coronary heart disease (CHD), peripheral arterial disease (PAD), tobacco-related carcinoma (lung, urinary tract or ENT) and COPD - was obtained from the patient's interview and from the past and current medical records, classified according to the International Classification of Diseases, 10th revision (ICD-10) ${ }^{13}$ COPD was also assessed on lung function when $\mathrm{FEV}_{1} / \mathrm{FVC}$ was less than $70 \%{ }^{14}$ We defined coronary heart disease (CHD) as a history of angina pectoris, myocardial infarction, coronary arterial bypass graft, arterial angioplasty or known vessel obstruction of $50 \%$ or more. Leg pain associated with an ankle brachial ratio index less than 0.9 or a procedure for arterial insufficiency defined a peripheral arterial disease (PAD). We recorded coughing, sputum production and the degree of dyspnoea according to the New York Heart Association (NYHA) classification. ${ }^{15}$

\section{Factors for stains development}

We documented any mental disorders diagnosed according to the Diagnostic and Statistical Manual of Mental Disorders (DSM-IV) criteria and antidepressant, benzodiazepine, neuroleptic or mood stabiliser use. ${ }^{16}$ We considered harmful alcohol use when high alcohol intakemore than 14 standard drinks/week for women and more than 21 standard drinks/week for men (140 and $210 \mathrm{~g}$ of alcohol, respectively) —was associated with documented withdrawal, medical consequences 
(neurological, cardiac, hepatic or pancreatic) or erythrocyte macrocytosis (mean corpuscular volume (MCV) greater than $98 \mathrm{fL}$ ) not otherwise explained. ${ }^{17}$

Occupations were classified according to the six-class version of the National Statistics Socio-Economic Classification. ${ }^{18}$ Previous occupation was considered for retired individuals. Smoking habits reviewed were: age at onset, number of packets smoked per day, unfiltered or low tar cigarettes and attempts at smoking cessation.

Patients were questioned on the number of years the stains have been present, their tendency to extend, their impact on everyday life, and attempts to make them disappear. We recorded the number and location of stains for each patient as well as the surface area in centimetres squared of the stain located on the finger skin only. The intensity of all stains was graded using a standardised visual scale from 1 to 5 depending on the colour (pale yellow to intense brown).

\section{Statistical analysis}

We paired one control for every case, and matched them individually by age, gender, height and pack-years (PYs) smoked. Pairs were matched closely with up to $10 \%$ difference for each continuous variable. We then compared the predicted value of $\mathrm{FEV}_{1}$ (according to height, age and gender) of each pair to ensure a difference of $<10 \% .{ }^{19}$ The PY was calculated as the number of packs smoked in a day multiplied by the number of years spent smoking. When the consumption was irregular from day to day, we used the mean of the extremes as an estimate of the number of packs smoked daily. If the consumption was higher or lower for more than a year's duration, the total cumulative PY was calculated as the sum of the different PYs throughout life.

We calculated that 50 participants in each group would allow us to detect a threefold increase of tobacco-related disease in the cases group (OR), using the McNemar test with a two-sided $\alpha$ of $5 \%$ and a power of $80 \%$, if at least $30 \%$ of pairs are discordant. To compare groups, we used the McNemar test for binary variables and paired Wilcoxon test for continuous variables. Among the case group, we used Spearman's rank correlation to test for an association between the measured $\mathrm{FEV}_{1}$ and stain characteristics.

Conditional logistic regression was used to assess the association between cigarette staining and mental health condition, psychotropic medications, harmful alcohol use, occupational categories and smoking behaviours. We built a parsimonious multivariate model, guided by the analyst, entering each potential variable one by one, starting with those strongly associated and clinically relevant and keeping only statistically significant and mutually adjusted variables in the final model. With about 50 events (cases) and a requested number of 10-15 events per variable, we expected a maximum of 4-5 variables in the final. ${ }^{20}$

We performed post hoc subanalyses excluding pairs in which controls notified finger/nail stains in the past.
Since those analyses showed similar results, only results of the initial analyses are shown.

Analyses were performed on cases and controls with complete documented information. Data analyses were performed by using STATA V.10.1 (StataCorp LP, Texas, USA). This study received no external funding source and volunteers received no financial reward.

\section{RESULTS}

\section{Participants}

Between March 2006 and December 2007, 4520 patients were admitted to the hospital, of which 1127 were current smokers $(25 \%)$. Among those current smokers, $63(6 \%)$ presented one or more tar staining on the hand. Nine refused to participate and five could not be matched, resulting in 49 cases. The corresponding 49 controls were included between February 2007 and January 2010. All participants were Caucasians (table 1).

For one participant, the stain characteristics were impossible to determine due to a peripheral cyanosis at the time of examination. The 48 other cases possessed a total of 153 stains, $64 \%$ on the nail and $36 \%$ on the finger skin (table 2). Among the controls, 11 (22\%) reported the presence of at least one stain on the fingers within their lifetime but not at the time of the study.

\section{Tobacco-related disease and lung function test}

Although there was a slight increase in vascular disease, COPD and smoking-related cancer in the case group, the difference was not statistically significant (table 3). PAD was more prevalent among the case group with 14/ $49(29 \%)$ presenting with claudication and $12 / 49(24 \%)$ having undergone revascularisation procedure compared to $5 / 49(10 \% ; \mathrm{p}=0.049)$ and $2 / 49(4 \% ; \mathrm{p}=0.006)$, respectively, in the control group.

Lung function tests were not available for three controls. Measured $\mathrm{FEV}_{1}$ was not statistically different between groups. Thirteen cases $(26 \%)$ and 11 of 46 (24\%) controls had less than $50 \%$ of the expected $\mathrm{FEV}_{1}$. Stain number ( $\mathrm{r}: 0.13, \mathrm{p}=0.39)$, mean size $(\mathrm{r}: 0.24$, $\mathrm{p}=0.25)$ and median colour intensity $(\mathrm{r}: 0.22, \mathrm{p}=0.13$ ) had no influence on measured $\mathrm{FEV}_{1}$ among cases.

\section{Factors associated with tar staining}

Smoking more than one pack of cigarettes per day, smoking unfiltered cigarettes, having more cigarette breaks, harmful alcohol use or being on psychotropic medication were all associated with a higher risk of tar staining in univariate analyses (table 4). Use of low tar content cigarettes was less frequent among tobacco stain participants.

In a multivariate analysis, four factors remained significantly associated with stain: smoking more than 1 pack/ day, smoking unfiltered cigarettes, age at onset and harmful alcohol use. Adding more variables produced an artificially high OR due to the insufficient 
Table 1 Baseline characteristics of the yellow-stained finger cases and of control smokers

\begin{tabular}{|c|c|c|c|}
\hline Variables & Cases $(n=49)$ & Controls $(n=49)$ & p Value* \\
\hline \multicolumn{4}{|l|}{ Matching } \\
\hline Median (IQR) age (years) & $62(50-69)$ & $59(50-69)$ & $0.65 \dagger$ \\
\hline Male & $32(65)$ & $32(65)$ & 0.99 \\
\hline Median (IQR) height (cm) & $170(165-177)$ & $170(165-175)$ & $0.84 \dagger$ \\
\hline Median (IQR) pack-years smoked $\ddagger$ & $50(40-61)$ & $46(38-58)$ & $0.20 \dagger$ \\
\hline Median (IQR) predicted FEV ${ }_{1}(\mathrm{~L}) \S$ & $3.3(2.4-3.8)$ & $3.2(2.4-3.7)$ & $0.60 \dagger$ \\
\hline \multicolumn{4}{|l|}{ Diagnosis category on admissionๆ } \\
\hline Cardiovascular & $11(22)$ & $15(31)$ & 0.48 \\
\hline Neurological & $7(14)$ & $6(12)$ & 0.99 \\
\hline Oncology & $8(16)$ & $6(12)$ & 0.79 \\
\hline Infectious disease & $5(10)$ & $5(10)$ & 0.99 \\
\hline Traumatology & $6(12)$ & $5(10)$ & 0.99 \\
\hline Others & $12(24)$ & $12(24)$ & 0.99 \\
\hline
\end{tabular}

Values are numbers (percentage) unless stated otherwise.

IQR 25-75\%.

${ }^{*}$ McNemar and exact test unless stated otherwise.

†Paired Wilcoxon sign-rank test.

$\ddagger$ Number of packs smoked in a day multiplied by the number of years spent smoking or sum of the different PYs throughout life if the

consumption was higher or lower for more than a year's duration.

§Predicted FEV1 calculated according to the third National Health and Nutrition Examination Survey formula. ${ }^{19}$

१Category of main diagnosis claimed on the exit chart.

$\mathrm{FEV}_{1}$, maximal forced expiratory volume in $1 \mathrm{~s}$; PY, pack-year unit.

events-per-variable. These four factors accounted for $41 \%$ of the variance (pseudo-R2) in the presence of stains.

Table 2 Stains characteristics in cases

\begin{tabular}{lc}
\hline Stains characteristics & Cases $\mathbf{N}(\%)^{\star}$ \\
\hline Time of stain appearance (years) & $15(31)$ \\
$1-4$ & $2(4)$ \\
$5-9$ & $31(65)$ \\
$\geq 10$ & $3(6)$ \\
Suffering due to the stain & $23(48)$ \\
Physical/chemical attempt to remove the & \\
stain & $18(37)$ \\
Variation over time (stain size or intensity) & $3(2-4)$ \\
Median (IQR) stains per cases & $2(1-3)$ \\
$\quad$ Nails stained & $1(0-2)$ \\
Stains on skin & $3(2-4)$ \\
Median (IQR) number of fingers & \\
discoloured per case & $25(52)$ \\
First finger & $45(94)$ \\
Second finger & $37(77)$ \\
Third finger & $1(2)$ \\
Fourth finger & $0(0)$ \\
Fifth finger & $1.9(1.0-3.1)$ \\
Median (IQR) total skin area stained per & \\
case (square centimetre) & \\
\hline Values are numbers of cases (percentage) unless stated \\
otherwise. \\
IQR 25-75\%. \\
*Stain location, size and colour intensity were impossible to \\
determine for one patient due to peripheral cyanosis at the time of \\
examination. In another three cases, the precise size of stains \\
present on the skin (five stains) was not measured because of \\
scant demarcation of the stain. \\
$\quad$ \\
\hline
\end{tabular}

\section{DISCUSSION}

Our case-control study explores an easily identifiable and frequent clinical sign among smokers. Tobaccorelated diseases (defined as smoking-related carcinoma, ischaemic heart disease, PAD, stroke and COPD) are frequent $(84 \%)$ among patients with tobacco-tar-stained fingers. Furthermore, this clinical sign is linked to harmful alcohol use. As far as we know, this is the first description of this particular category of smokers.

The design of the study appreciates the additional risk for health due to tar deposition not being included in the usual measure of tobacco exposure (PY). Stain may result from local pigment deposition secondary to high-tar concentration in the skin-cigarette interface (influenced by puffing or cigarette characteristics), but a higher concentration of nicotine (and other substances) smoked could affect stain removal by impaired skin repair. ${ }^{21}$ Similarly, nicotine has been shown to reduce blood flow in fingers and increase systemic blood pressure, a mechanism that could rationally explain the association of impaired stain removal and PAD. ${ }^{22} 23$

Yet the high incidence of COPD, overall cardiovascular disease and tobacco-related carcinomas is not independently related to tar deposition. Besides, similar lung function between case and control groups and the absence of relationship between $\mathrm{FEV}_{1}$ and stain characteristics among cases are consistent with a lack of association between yellow fingers and obstructive lung disease. We believe that yellow staining identifies highrisk smokers due to the tobacco exposure (PY), rather than a specific increased susceptibility (eg, puffing characteristics). Lack of an association with investigated tobacco-related illnesses other than symptomatic PAD 
Table 3 Tobacco-related diseases and respiratory discomforts

\begin{tabular}{|c|c|c|c|c|c|}
\hline & Cases & Controls & $\mathrm{N}$ pairs & OR $(95 \% \mathrm{Cl})$ & p Value* \\
\hline \multicolumn{6}{|l|}{ Tobacco-related disease } \\
\hline Any tobacco-related disease $\dagger$ & $41(84)$ & $39(80)$ & 49 & $1.3(0.4$ to 4.1$)$ & 0.80 \\
\hline Vascular disease & $25(51)$ & $26(53)$ & 49 & $0.9(0.4$ to 2.3$)$ & 0.83 \\
\hline Coronary arterial disease & $17(35)$ & 19 (39) & 49 & $0.8(0.3$ to 2.1$)$ & 0.67 \\
\hline Strokes/TIA§ & $4(8)$ & $6(12)$ & 49 & $0.5(0.1$ to 3.5$)$ & 0.69 \\
\hline Peripheral arterial diseaseף & $15(31)$ & $5(10)$ & 49 & 3.5 (1.1 to 14.6$)$ & 0.03 \\
\hline COPD & $30(64)$ & $24(49)$ & 47 & $2.2(0.7$ to 8.1$)$ & 0.13 \\
\hline $\mathrm{FEV}_{1} / \mathrm{FVC}<70 \%$ & $24(52)$ & $17(37)$ & 46 & $2.2(0.8$ to 6.9$)$ & 0.11 \\
\hline Clinical diagnosis** & $22(45)$ & $20(41)$ & 49 & $1.3(0.4$ to 4.7$)$ & 0.59 \\
\hline Smoking-related cancer†† & $10(20)$ & $7(14)$ & 49 & $1.5(0.5$ to 5.1$)$ & 0.61 \\
\hline \multicolumn{6}{|l|}{ Respiratory discomforts } \\
\hline Coughing & $32(65)$ & $25(51)$ & 49 & 2.2 (0.8 to 6.9$)$ & 0.11 \\
\hline Sputum production & 19 (39) & $15(31)$ & 49 & $1.8(0.5$ to 6.8$)$ & 0.28 \\
\hline Median (IQR) grade dyspnoeał‡ & $1(1-2)$ & $1(0-2)$ & 49 & - & $0.14 \S \S$ \\
\hline Abnormal lung auscultationף & $24(49)$ & $10(20)$ & 49 & 4.5 (1.5 to 18.3$)$ & 0.004 \\
\hline Median (IQR) FEV F $_{1}(\mathrm{~L})$ & $1.9(1.3-2.5)$ & $1.8(1.4-2.6)$ & 46 & - & $0.49 \S \S$ \\
\hline
\end{tabular}

Values are numbers of cases (percentage) unless stated otherwise.

IQR, 25-75\%.

*McNemar exact test.

†At least one vascular disease, COPD or smoking-related carcinoma.

$\ddagger$ Self-reporting angina pectoris, heart infarction, coronary arterial bypass graft, percutaneous angioplasty or more than $50 \%$ vessel obstruction on coronary catheterisation.

§Stroke or a transient neurological symptom except a traumatic, haemorrhagic or cardioembolic event.

ๆSelf-reporting symptomatic arterial claudication, ankle brachial ratio less than 0.9 or chirurgical or radiological procedure for arterial

insufficiency.

${ }^{* *}$ Self-reporting or documented in the medical record.

††Carcinoma of the lung, urinary tract or head and neck.

$\ddagger \ddagger$ According to the New York Heart Association functional capacity classification.

$\S \S$ Paired Wilcoxon sign-rank test.

१ๆTheezing or crackles.

COPD, chronic obstructive pulmonary disease; $\mathrm{FEV}_{1}$, maximal forced expiratory volume in one second; FVC, forced vital capacity; $\mathrm{N}$ pairs,

number of pairs used in the analysis; TIA, transient ischaemic attack.

Table 4 Smoking behaviour, mental health, harmful alcohol use, and occupation categories in univariated and multivariated conditional regression models

\begin{tabular}{|c|c|c|c|c|c|c|}
\hline & \multirow[b]{2}{*}{ Cases } & \multirow[b]{2}{*}{ Controls } & \multicolumn{2}{|l|}{ Univariated } & \multicolumn{2}{|l|}{ Multivariated } \\
\hline & & & OR (Cl 95\%) & $p$ Value & aOR (Cl 95\%) & $p$ value \\
\hline \multicolumn{7}{|l|}{ Smoking habits } \\
\hline Median (IQR) age at beginning (years) & $17(15-20)$ & $18(15-20)$ & $1.5(1.0 \text { to } 2.4)^{\star}$ & 0.06 & $2.1(1.1 \text { to } 4.0)^{*}$ & 0.02 \\
\hline Median (IQR) number of packs per day & $1(1-1.8)$ & $1(1-1.5)$ & $3.9(1.2$ to 12.7$) \dagger$ & 0.02 & $8.5(1.9$ to 38.4$) \dagger$ & 0.006 \\
\hline $\mathrm{Nb}$ of brand median (IQR) & $2(1-3)$ & $2(2-3)$ & 0.9 (0.7 to 1.2$)$ & 0.39 & - & - \\
\hline Breaks median (IQR) & $1(0-2)$ & $2(1-3)$ & $0.7(0.5$ to 1$)$ & 0.03 & - & - \\
\hline $\mathrm{Nb}$ patients smoking unfiltered cigarettes & $20(41)$ & $9(18)$ & $3.2(1.2$ to 8.7$)$ & 0.02 & $4.2(1.1$ to 16.0$)$ & 0.04 \\
\hline $\mathrm{Nb}$ patients smoking low tar cigarettes & $7(14)$ & $17(35)$ & $0.3(0.1$ to 0.9$)$ & 0.03 & - & - \\
\hline \multicolumn{7}{|l|}{ Mental health and harmful alcohol use } \\
\hline Harmful alcohol use $\ddagger$ & $25(51)$ & $10(20)$ & $3.5(1.4$ to 8.7$)$ & 0.007 & $4.6(1.2$ to 17.2$)$ & 0.02 \\
\hline Other psychiatric condition§ & $22(45)$ & $14(29)$ & 1.7 (0.8 to 3.7$)$ & 0.18 & - & - \\
\hline On psychotropic medicationף & $33(67)$ & $20(41)$ & $2.4(1.1$ to 5.3$)$ & 0.02 & - & - \\
\hline \multicolumn{7}{|l|}{ Occupation } \\
\hline Unemployment & $17(35)$ & $12(24)$ & $1.7(0.7$ to 4.3$)$ & 0.26 & - & - \\
\hline
\end{tabular}

Values are numbers (percentage) unless stated otherwise.

*For every 2 years younger than 22 years.

†For every 10 cigarettes more than 20.

¥More than 14 drinks/week for women, more than 21 drinks/week for men associated with alcohol withdrawal or documented complications.

$\S$ According to the medical chart and DSM-IV criteria.

IAntidepressant, anxiolytic, neuroleptic and antiepileptic (used as mood stabiliser) drug.

aOR, adjusted OR; DSM-IV, Diagnostic and Statistical Manual of Mental Disorders. 
could however arise from the insufficient power of the study.

Four variables mutually adjusted-smoking more than one pack of cigarettes in a day or unfiltered cigarettes, age at smoking onset and harmful alcohol use-could explain a substantial part of stain development, even in individuals matched for characteristics that are probably linked to stains (pack-year, age and gender). Our results suggest that staining is influenced by the characteristics of the cigarettes smoked and the smoker's behaviour. Indeed, finger-stained smokers seem to present a more severe addictive behaviour (high proportion of harmful alcohol use, early age at smoking onset, high number of cigarettes smoked per day and few cigarette breaks). An industry-funded study concluded that psychological and personality constructs were positively associated with depth of inhalation. ${ }^{24}$ Apart from puffing characteristics, smoking the entirety of cigarettes, seen among addict individuals, might also contribute to tar staining. Previous studies have shown that the proportion of alcohol disorders increases with cigarette consumption, and that daily tobacco use increases with the level of alcohol consumption. ${ }^{25}$ Our study is the first to demonstrate an association between harmful alcohol use and tar staining independent of the extent of tobacco consumption. In itself, alcohol consumption could influence smoking behaviour, which in turn impacts on tar deposition. Nil and colleagues reported increased puff volume following the consumption of $0.7 \mathrm{~g} / \mathrm{kg}$ of ethanol. ${ }^{27} \mathrm{We}$ believe that this addictive behaviour and concomitant high alcohol consumption could impact tobacco cessation programmes, although it has not been tested yet.

The study has several limitations. First, many diagnoses were obtained from medical records or from patients self-reporting. Thus, the study was dependent on the quality and completeness of medical charts and subject to recall bias. This assessment might also cover a specific fraction of the mental disorders (those who seek professional help). Second, tobacco dependence or problematic alcohol consumption was not measured with established instruments (eg, an Alcohol Use Disorders Identification Test (AUDIT)). 'Harmful alcohol use' is not a standard definition and includes MCV, which has a low specificity. Furthermore, the observers were not blinded to the allocation (case vs control) of the patients, which could influence clinical interview. However, the results are plausible, and future studies should use standard tools to confirm them. Third, a power size calculation was made for 50 pairs and only 49 could be found because of the restrictive matching criteria. Moreover, 22\% of controls reported transient tar discoloration in the past, which might have blurred the effect. However, tar deposition appears to be constant over time in the case group, and a transient finger staining could be a clinically insignificant event. Finally, our observations are limited to the inpatient hospital setting and may have to be confirmed in finger-stained patients seen outside this context.
In a world of growing technology and complicated diagnosis procedure, our work highlights the importance of the clinical assessment of smokers, an important message to transmit in medical education. This costless and easily obtainable information should stress on tobacco-related disease screening and reinforce smoking counselling. Harmful alcohol use should be explored among these patients.

Acknowledgements The authors gratefully acknowledge Sebastian Carballo, Fraser Easton and Andrew Stewardson for their correction of the English manuscript.

Contributors All authors have participated in the conception and design or analysis and interpretation of the data, drafting of the article or in revising it critically for important intellectual content. GH was involved in the drafting of the manuscript. GJ and DG contributed to the study concept and design. They had full access to all of the data in the study and take responsibility for the integrity of the data and the accuracy of the data analysis. SP, NR, AC and GJ contributed to the acquisition of data. CD-C and GJ contributed to the analysis and interpretation of the data and statistical analysis. CD-C, SP, NR, $A C$ and $D G$ were involved in the critical revision of the manuscript for important intellectual content. DG was involved in the study supervision.

Funding This research received no specific grant from any funding agency in the public, commercial or not-for-profit sectors.

Competing interests None.

Patient consent Obtained.

Ethics approval Ethical board of the institution.

Provenance and peer review Not commissioned; externally peer reviewed.

Data sharing statement The full dataset can be obtained on request from the corresponding author.

Open Access This is an Open Access article distributed in accordance with the Creative Commons Attribution Non Commercial (CC BY-NC 3.0) license, which permits others to distribute, remix, adapt, build upon this work noncommercially, and license their derivative works on different terms, provided the original work is properly cited and the use is non-commercial. See: http:// creativecommons.org/licenses/by-nc/3.0/

\section{REFERENCES}

1. World Health Organziation. Report on the global tobacco epidemic. 2011. http://www.who.int/tobacco/global_report/2011/qanda/en/index. html

2. Ezzati M, Lopez AD. Regional, disease specific patterns of smoking-attributable mortality in 2000. Tob Control 2004;13:388-95.

3. United States. Public Health Service. Office of the Surgeon General. How tobacco smoke causes disease the biology and behavioral basis for smoking-attributable disease: a report of the Surgeon General. Rockville, MD, [Washington, D.C.]: U.S. Dept. of Health and Human Services, Public Health Service. For sale by the Supt. of Docs., U.S. G.P.O., 2010:1 online resource (xv, 706 p.).

4. Aberle DR, Adams AM, Berg CD, et al. Reduced lung-cancer mortality with low-dose computed tomographic screening. $N$ Engl J Med 2011;365:395-409.

5. Solly S. Clinical lectures on paralysis. Lancet 1856;63:669-71.

6. Lange $P$, Schnohr $P$. The relationship between facial wrinkling and airflow obstruction. Int J Dermatol 1994;33:123-6.

7. Levine $\mathrm{H}$. The yellow rounded digit: sign of the times. $N$ Engl J Med 1968;279:660-1.

8. Verghese A, Krish G, Howe D, et al. The harlequin nail. A marker for smoking cessation. Chest 1990;97:236-8.

9. Lawrence D, Mitrou F, Zubrick SR. Smoking and mental illness: results from population surveys in Australia and the United States. BMC Public Health 2009;9:285.

10. Kalman D, Morissette SB, George TP. Co-morbidity of smoking in patients with psychiatric and substance use disorders. Am J Addict 2005;14:106-23.

11. Thakurdas H. Smoking and psychiatrc patient. BMJ 1963;2:388. 
12. Hafezi M, Bohnert $M$, Weinmann $W$, et al. Prevalence of nicotine consumption in drug deaths. Forensic Sci Int 2001;119:284-9.

13. World Health Organisation. International statistical classification of diseases and related health problems. 10th edn. Geneva: World Health Organisation, 2007

14. Global Initiative for Chronic Obstructive Lung Disease (GOLD) Global Strategy for the Diagnosis, Management, and Prevention of Chronic Obstructive Pulmonary Disease. 2009.

15. The Criteria Committee of the New York Heart Association. Nomenclature and criteria for dagnosis of diseases of the heart and great vessels. 9th edn. Boston: The Criteria Committee of the New York Heart Association, 1994.

16. American Psychiatric Association. Diagnostic and Statistical Manual of Mental Disorders. 4th edn. Washington, DC: American Psychiatric Association, 1994.

17. World Health Organziation. Lexicon of alcohol and drug terms published by the World Health Organization. 2005. http://www.who. int/substance_abuse/terminology/who_lexicon/en/

18. The National Statistics Socio-economic Classification (NS-SEC rebased on the SOC2010), 2010.

19. Hankinson JL, Odencrantz JR, Fedan KB. Spirometric reference values from a sample of the general U.S. population. Am J Respir Crit Care Med 1999;159:179-87.
20. Courvoisier DS, Combescure C, Agoritsas T, et al. Performance of logistic regression modeling: beyond the number of events per variable, the role of data structure. J Clin Epidemiol 2011;64:993-1000.

21. Sorensen LT, Toft B, Rygaard J, et al. Smoking attenuates wound inflammation and proliferation while smoking cessation restores inflammation but not proliferation. Wound Repair Regen 2010;18:186-92.

22. Reus WF, Robson MC, Zachary L, et al. Acute effects of tobacco smoking on blood flow in the cutaneous micro-circulation. $\mathrm{Br} \mathrm{J}$ Plast Surg 1984;37:213-15

23. Rojanapongpun $\mathrm{P}$, Drance SM. The effects of nicotine on the blood flow of the ophthalmic artery and the finger circulation. Graefes Arch Clin Exp Ophthalmol 1993;231:371-4.

24. Wood D, Wilkes E. Project Wheat-Part 1 Cluster Profiles of U.K Male Smokers and Their General Smoking Habits. Report No. Rd.1229-R., 1960.

25. Friedman GD, Tekawa I, Klatsky AL, et al. Alcohol drinking and cigarette smoking: an exploration of the association in middle-aged men and women. Drug Alcohol Depend 1991;27:283-90.

26. Dawson DA. Drinking as a risk factor for sustained smoking. Drug Alcohol Depend 2000;59:235-49.

27. Nil R, Buzzi R, Battig K. Effects of single doses of alcohol and caffeine on cigarette smoke puffing behavior. Pharmacol Biochem Behav 1984;20:583-90. 